\title{
Effective Hexagonal Boron Nitride Passivation of Few-Layered InSe and GaSe to Enhance Their Electronic and Optical Properties
}

\author{
Himani Arora, ${ }^{\dagger, \uparrow 0}$ Younghun Jung, ${ }^{\S}$ Tommaso Venanzi, ${ }^{\dagger,+}$ Kenji Watanabe, $"$ Takashi Taniguchi,"

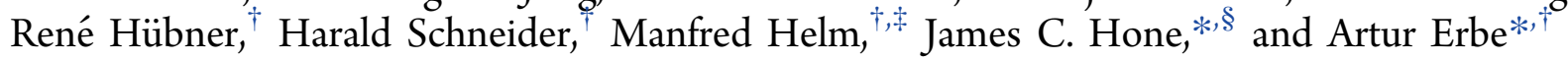 \\ ${ }^{\dagger}$ Helmholtz-Zentrum Dresden-Rossendorf, 01328 Dresden, Saxony, Germany \\ ${ }^{\ddagger}$ Technische Universität Dresden, 01062 Dresden, Saxony, Germany \\ ${ }^{\S}$ Department of Mechanical Engineering, Columbia University, 10027 New York, New York, United States \\ "National Institute for Materials Science, 1-1 Namiki, 305-0044 Tsukuba, Ibaraki, Japan
}

\author{
Supporting Information
}

ABSTRACT: Indium selenide (InSe) and gallium selenide (GaSe), members of the III-VI chalcogenide family, are emerging two-dimensional (2D) semiconductors with appealing electronic properties. However, their devices are still lagging behind because of their sensitivity to air and device fabrication processes which induce structural damage and hamper their intrinsic properties. Thus, in order to obtain high-performance and stable devices, effective passivation of these air-sensitive materials is strongly required. Here, we demonstrate a hexagonal boron nitride (hBN)-based encapsulation technique, where 2D layers of InSe and GaSe are covered entirely between two layers of hBN. To fabricate devices out of fully encapsulated 2D layers, we employ the lithography-free via-contacting scheme. We find that hBN acts as an excellent encapsulant and a near-ideal substrate for InSe and GaSe by passivating them from the environment and isolating them from the charge disorder at the $\mathrm{SiO}_{2}$ surface. As a result, the encapsulated InSe devices are of high quality and ambient-stable for a long time and show an improved two-terminal mobility of $30-120 \mathrm{~cm}^{2} \mathrm{~V}^{-1} \mathrm{~s}^{-1}$ as compared to mere $\sim 1 \mathrm{~cm}^{2} \mathrm{~V}^{-1} \mathrm{~s}^{-1}$ for unencapsulated devices. On employing this technique to GaSe, we obtain a strong and reproducible photoresponse. In contrast to previous studies, where either good performance or long-term stability was achieved, we demonstrate a combination of both in our devices. This work thus provides a systematic study of fully encapsulated devices based on InSe and GaSe, which has not been reported until now. We believe that this technique can open ways for fundamental studies as well as toward the integration of these materials in technological applications.

KEYWORDS: indium selenide, gallium selenide, hexagonal boron nitride, encapsulation, photoluminescence, stable electronics, field-effect transistors, photodetectors
$\mathrm{T}$ he III-VI chalcogenide family ( $\mathrm{MX} ; \mathrm{M}=\mathrm{Ga}$, In and $\mathrm{X}=$ $\mathrm{S}, \mathrm{Se}, \mathrm{Te})$ has attracted considerable attention in recent years owing to its interesting properties and underlying physics at low dimensions. The two most prominent members of this family are indium selenide (InSe) and gallium selenide ( $\mathrm{GaSe}$ ), which are promising candidates for various applications such as high-speed electronics, ${ }^{1-4}$ optoelectronics, ${ }^{5-10}$ sensors, ${ }^{11-13}$ and terahertz technology. ${ }^{14}$ Recently, devices based on InSe have seen many advancements owing to its high electron mobility ${ }^{1-3}$ resulting from its low electron effective mass $\left(m_{\mathrm{e}}^{*}\right.$ $\left.=0.143 m_{\mathrm{o}}\right)^{15}$ and a direct band gap lying in the near-infrared range. ${ }^{16}$ The electron mobility of $\sim 1000 \mathrm{~cm}^{2} \mathrm{~V}^{-1} \mathrm{~s}^{-1}$ obtained at room temperature ${ }^{1-3}$ in InSe devices is the highest value reported for an n-type two-dimensional (2D) material so far. $\mathrm{GaSe}$, on the other hand, is a p-type material with a band gap of $2.11 \mathrm{eV}$ in bulk and well known for its optical properties. ${ }^{17-20}$ In terms of electronic transport, few-layered GaSe has revealed mobilities of $0.1-0.6 \mathrm{~cm}^{2} \mathrm{~V}^{-1} \mathrm{~s}^{-1}$ when integrated as a channel into field-effect transistors (FETs). ${ }^{8,11,20}$ Unlike InSe, GaSe is not a high-mobility material due to the presence of heavy holes, but it is still very appealing for optoelectronics, ${ }^{8,21,22}$ single-photon emission, ${ }^{23}$ nonlinear optics, ${ }^{24}$ and terahertz applications. ${ }^{14}$ Despite many promising properties of InSe and GaSe, limited work has been done exploiting them into devices because of their instability under ambient conditions.

Though GaSe has been demonstrated to degrade rapidly on interaction with ambient air, ${ }^{21,25-27}$ the question whether InSe is air-sensitive or not is yet unclear within the scientific community. Some reports have claimed it to be relatively stable with no observable degradation over time $e^{4,26,28,29}$ and extracted high mobilities under normal atmospheric con-

Received: July 29, 2019

Accepted: October 25, 2019

Published: October 25, 2019 
ditions, ${ }^{2,3}$ while others observed that it degrades on contact with water and oxygen. ${ }^{30-32}$ Even standard process steps of device fabrication involving lithographic patterning, resist spinning, and solvents' dips can cause considerable structural damage, degrading the quality of the material and resulting in poor performance of the devices. ${ }^{7,33}$ It is worth mentioning that most previous reports which demonstrated high mobility on bare InSe used thicker layers $(>30 \mathrm{~nm})$ and shadow masks to fabricate their devices. ${ }^{2-5}$ However, as InSe layer thickness approaches the quantum confinement limit, it becomes increasingly sensitive to environmental influences such as oxygen, moisture, charge traps in the dielectric and contaminations from chemical solvents, leading to fast and uncontrollable morphological changes and poor device performance. Therefore, in order to obtain high-performance and stable devices based on these materials, effective encapsulation techniques should be developed to protect InSe and $\mathrm{GaSe}$ layers against degradation.

Recently, the techniques of dry oxidation ${ }^{32}$ and seeded atomic layer deposition (ALD) ${ }^{34}$ have been demonstrated to overcome the instability of InSe. While both techniques use oxide-based encapsulation, they provide only top encapsulation, whereas the bottom surface of InSe rests on a $\mathrm{SiO}_{2}$ substrate. $\mathrm{SiO}_{2}$ has been revealed to be an unsuitable dielectric for $2 \mathrm{D}$ materials as it damages the material's quality and device performance by inducing the charge disorder at the interface due to scattering from charge traps, impurities, and surface roughness. $^{35,36}$ InSe-based FETs have previously shown substantial hysteresis and unreliability in their $\mathrm{I}-\mathrm{V}$ characteristics due to charge trapping at the $\mathrm{InSe}-\mathrm{SiO}_{2}$ interface and hydration on the $\mathrm{SiO}_{2}$ surface. ${ }^{2}$ Moreover, ALD encapsulation requires lithography to fabricate devices, which as discussed above leads to further unreliability of the devices. For GaSe, hexagonal boron nitride (hBN) as top encapsulation has been recently reported, with a focus on its optoelectronic properties. $^{21}$ Though ambient-stable GaSe photodetectors were achieved, the performance was inferior to those reported on the $\mathrm{SiO}_{2}$ substrate. $8,22,37$

Here, we report a hBN-based encapsulation, where 2D layers of InSe and GaSe are sandwiched between two layers of hBN; top $\mathrm{hBN}$ passivating the $2 \mathrm{D}$ layer from the environment and bottom $\mathrm{hBN}$ acting as a spacer and suppressing charge transfer to the 2D layer from the $\mathrm{SiO}_{2}$ substrate. To fabricate the devices from fully encapsulated InSe and GaSe layers, we employ the technique of lithography-free via-contacts, ${ }^{38}$ which are metal contacts embedded within hBN flakes and allow us to simultaneously achieve encapsulation and an electrical connection to the underlying 2D layer without any direct lithographic patterning. Based on our results, we find that full $\mathrm{hBN}$ encapsulation preserves InSe in its pristine form and suppresses its degradation with time under ambient conditions. Consequently, the electronic properties of encapsulated InSe are significantly improved, leading to a two-terminal field-effect mobility $\left(\mu_{\mathrm{FE}}\right)$ ranging $30-120 \mathrm{~cm}^{2} \mathrm{~V}^{-1} \mathrm{~s}^{-1}$ and an on/off ratio of $10^{4}$ at room temperature as compared to $\mu_{\mathrm{FE}}$ of mere $\sim 1$ $\mathrm{cm}^{2} \mathrm{~V}^{-1} \mathrm{~s}^{-1}$ obtained for unencapsulated devices. In addition, encapsulated InSe devices are stable for a prolonged period of time, overcoming their limitation to be air-sensitive. On employing full hBN encapsulation to GaSe, photoluminescence (PL) is dramatically improved and a high photoresponsivity of $84.2 \mathrm{~A} \mathrm{~W}^{-1}$ at $405 \mathrm{~nm}$ is obtained.

This work advances previous efforts to encapsulate 2D layers of InSe and GaSe using hBN. The full hBN encapsulation technique passivates the air-sensitive layers from various degrading factors and preserves their unaltered properties. While previous studies have reported either good performance or long-term stability, we demonstrate a combination of both in our devices. The encapsulation technique we employ is robust and easily transferrable to other complex 2D materials which might not be compatible to the chemistry of ALD and dry oxidation techniques. Thus, this method opens up new avenues for the investigation of $2 \mathrm{D}$ materials which have been restricted so far in their fundamental study and applications due to environmental sensitivity.

\section{EXPERIMENTAL SECTION}

The hBN/2D/hBN (here 2D: InSe and GaSe) heterostructures are fabricated by using the van der Waals (vdW) transfer technique as described by Wang et al. ${ }^{39}$ In order to avoid exposing the $2 \mathrm{D}$ layers to air, the process steps from exfoliation of its bulk crystal until the $2 \mathrm{D}$ layers are fully covered between top and bottom $\mathrm{hBN}$ are performed inside a nitrogen-filled glovebox. Via-contacts used for the devices are fabricated as shown in the schematic of Figure 1a. hBN flakes $(30-40$
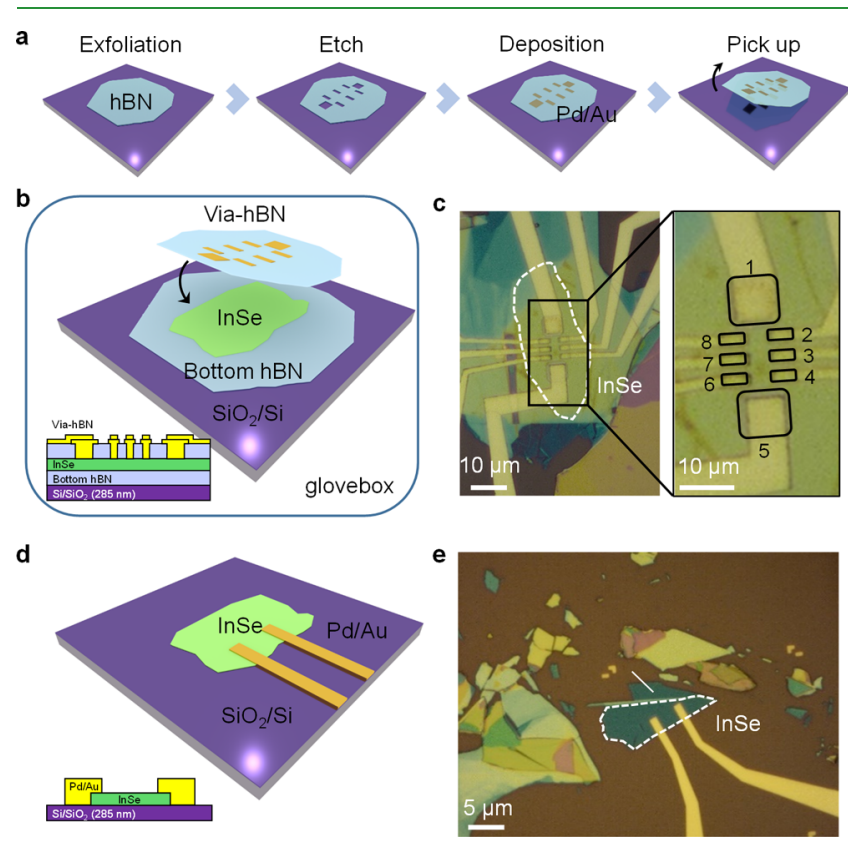

Figure 1. Fabrication of $\mathrm{Pd} / \mathrm{Au}$ via-contacts and two configurations of InSe-based device geometries investigated in this work. (a) Step-wise illustration of the via-contact fabrication process. (b) Schematic of the InSe-via device when the via-contact hBN covers InSe in a glovebox to encapsulate it and to form an electrical connection. (c) Optical image of the InSe-via device measured in this work. $9.6 \mathrm{~nm}$-thick InSe flake (white dashed line) is sandwiched between bottom hBN and top via-hBN. The black solid lines in the enlarged image highlight multiple $\mathrm{Pd} / \mathrm{Au}$ metal contacts (numbered 1-8) embedded in $\mathrm{hBN}$, which form a vdW contact with the underlying InSe layer. (d,e) Schematic and optical image of an unencapsulated device, respectively, white dashed line indicates the InSe flake on the $\mathrm{SiO}_{2}$ substrate and metal electrodes fabricated by depositing $\mathrm{Pd} / \mathrm{Au}$ metal.

$\mathrm{nm}$ thick, crack- and residue-free) exfoliated onto a $285 \mathrm{~nm} \mathrm{SiO} / 2 \mathrm{Si}$ substrate are identified using optical contrast and subsequently etched into desired electrode patterns using reactive-ion etching. The etched holes are then filled with $20 \mathrm{~nm}$ palladium $(\mathrm{Pd}) / 40 \mathrm{~nm}$ gold $(\mathrm{Au})$ by electron-beam evaporation. The via-contact is then picked up (Figure S1) and laminated onto the targeted $2 \mathrm{D}$ flake resting on a bottom $\mathrm{hBN}$ in a way that all via-metal electrodes cover the flake (Figure $1 \mathrm{~b}$ ). After full encapsulation, the whole assembly is moved out of the 
glovebox for fabricating metal extensions to the via-metals and contact pads, where the probes can be placed to carry out electrical measurements (see Methods and Supporting Information for details on the fabrication process). Figure $1 \mathrm{~b}, \mathrm{c}$ shows the schematic and the optical micrograph of one such InSe-via device, respectively, where black solid lines (in the enlarged image of Figure 1c) show the viametal in intimate contact with the underlying InSe layer. The heterostructures are fabricated in a contamination-free environment, so that the layers have clean and impurity-free interfaces. Having multiple via-contacts in one hBN flake allows us to contact and measure multiple devices on one single InSe flake. Figure $1 \mathrm{~d}$,e shows the schematic and the optical image of an unencapsulated InSe device, respectively, fabricated by electron beam lithography and metal deposition on InSe layers exfoliated directly onto a $285 \mathrm{~nm} \mathrm{SiO} 2 / \mathrm{Si}$ substrate. For a consistent comparison, both encapsulated and unencapsulated devices are fabricated with the same thickness of InSe that is $\sim 9.6 \mathrm{~nm}$ or 12 layers (confirmed with atomic force microscopy, shown in Figure S2) and the same configuration of the metal contacts (Pd: $20 \mathrm{~nm} / \mathrm{Au}: 40 \mathrm{~nm}$ ) deposited by electron-beam evaporation.

\section{RESULTS AND DISCUSSION}

The properties of the exfoliated thin layers depend strongly on the quality and composition of the bulk crystal. Figure $2 a, b$

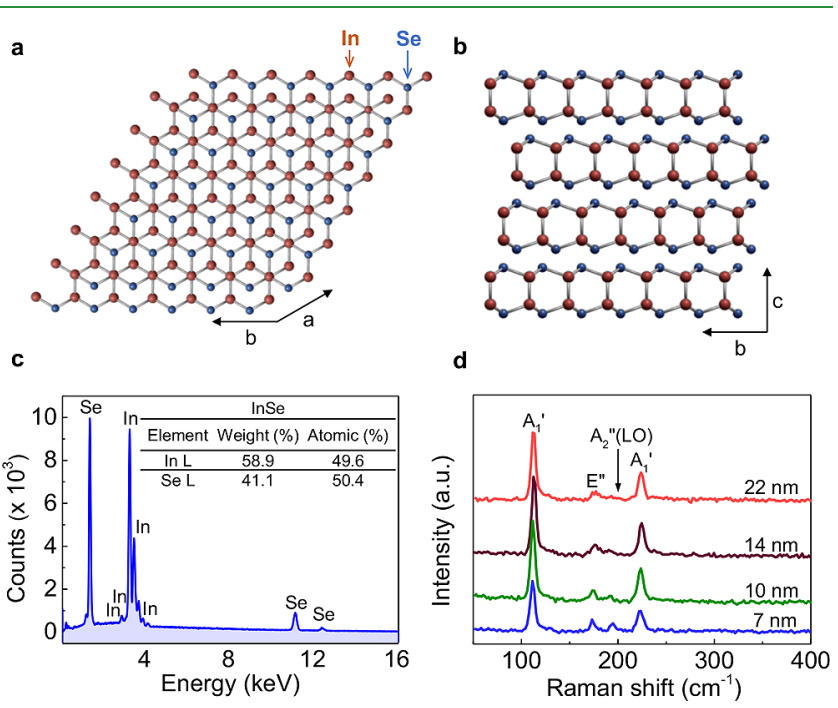

Figure 2. (a) Schematic of the top view of few-layered InSe showing the hexagonal lattice structure. (b) Side view of few-layered InSe showing the ABC stacking pattern of the $\gamma$-phase InSe (red balls: indium atoms, blue: selenium atoms). (c) EDS spectrum and compositional analysis of the InSe bulk crystal confirming an impurity-free crystal. (d) Raman spectra measured for various thicknesses of InSe at room temperature.

illustrates the schematics of top and side views of few-layered InSe. Energy-dispersive X-ray spectroscopy (EDS) and Raman spectroscopy are used to characterize the bulk and few layers of both InSe and GaSe. Figure $2 \mathrm{c}$ presents the EDS spectrum and compositional analysis of InSe bulk. The spectrum shows only indium and selenium peaks without any traces of impurities, which confirms high purity of the InSe crystals. The atomic ratio of $\mathrm{In} / \mathrm{Se}$ is found to be $1: 1$. The Raman spectra measured at room temperature for various thicknesses ranging 7-22 nm of InSe are shown in Figure 2d. In all spectra, four major vibrational modes are recognized which are consistent with previously reported Raman spectra on thin films of InSe. ${ }^{26,40}$ For the $10 \mathrm{~nm}$ thick InSe layer, the peaks at 113 and $223 \mathrm{~cm}^{-1}$ correspond to the nonpolar $A_{1}$ mode and those at 176 and 193 $\mathrm{cm}^{-1}$ to the polar $\mathrm{E}$ and $\mathrm{A}_{2}$ (LO) modes, which are typical modes for $\gamma$-InSe. For GaSe, the EDS and Raman spectra are measured as well (Figure S3). The EDS analysis confirms that the crystal is impurity-free, while the Raman spectrum shows five prominent peaks at 57.3, 132.8, 211.5, 245, and 307.1 $\mathrm{cm}^{-1}$ which are characteristic Raman modes for pristine $2 \mathrm{H}$ $\mathrm{GaSe}^{20,22,27,41,42}$

To demonstrate the effect of full hBN encapsulation on the stability of InSe, we employed micro-PL measurements to adjacent surfaces of exposed and protected InSe. Figure $3 \mathrm{a}, \mathrm{b}$
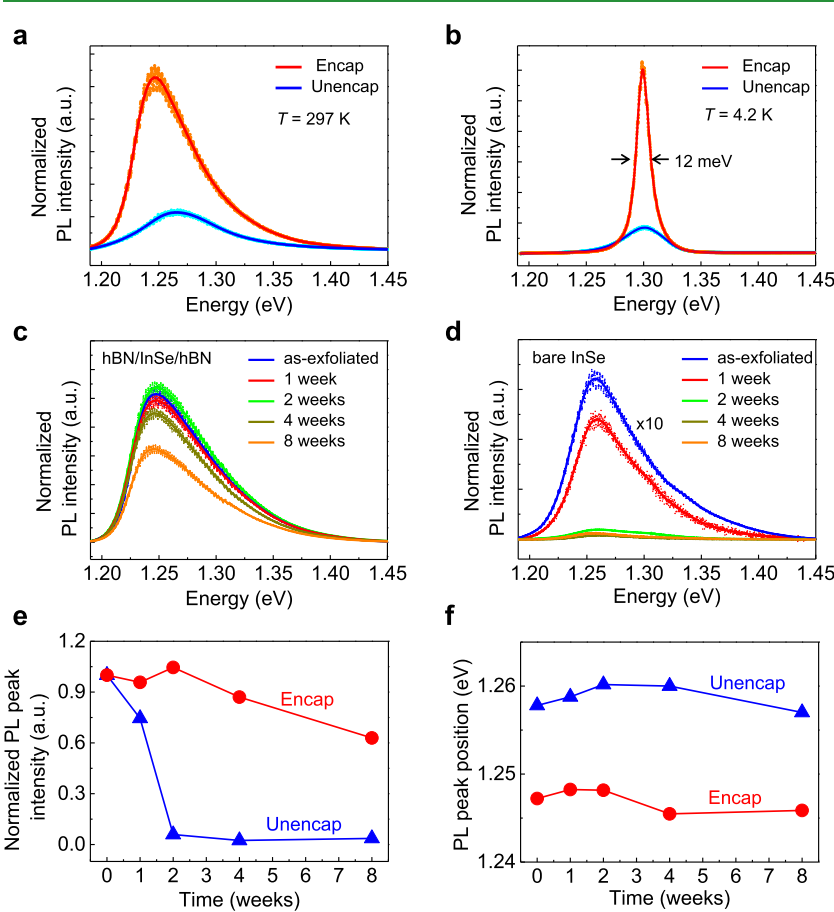

Figure 3. PL spectra measured for encapsulated and unencapsulated InSe, demonstrating high quality and ambient stability of fully hBNencapsulated InSe. PL spectra measured for both samples, (a) at 297 $\mathrm{K}$, showing higher intensity PL peak, and (b) at $4.2 \mathrm{~K}$, showing a narrower linewidth with fwhm of $12 \mathrm{meV}$ for encapsulated InSe. (c,d) Evolution of the PL spectra with time for both encapsulated and unencapsulated InSe at room temperature, respectively, stored under ambient conditions. $(\mathrm{e}, \mathrm{f})$ Normalized PL peak intensity and PL peak position measured over time, respectively, showing structural instability of InSe when exposed to air, whereas the encapsulated sample stays intact over 4 weeks.

shows the PL spectra obtained for both samples at 297 and 4.2 $\mathrm{K}$, respectively. It is evident that at both temperatures, $\mathrm{hBN}$ encapsulation significantly enhances the PL spectra by yielding narrower and more intense peaks as compared to the unencapsulated InSe due to crystalline and atomically flat layers of hBN. The prominent effect of hBN encapsulation is seen at $4.2 \mathrm{~K}$ (Figure $3 \mathrm{~b}$ ), where the PL linewidth narrows down to give a full width at half maximum (fwhm) of $\sim 12$ $\mathrm{meV}$ in contrast to $36-38 \mathrm{meV}$ obtained for the exposed InSe of the same thickness. The reduction of the PL linewidth and improved PL yield in the encapsulated sample indicates fabrication of a high-quality $\mathrm{hBN} / \mathrm{InSe} / \mathrm{hBN}$ vdW heterostructure. As compared to the $\mathrm{PL}$ at $4.2 \mathrm{~K}$, the linewidth of both samples broadens at $297 \mathrm{~K}$ and shows a red shift (approx. $50 \mathrm{meV}$ ) from 1.30 (at $4.2 \mathrm{~K}$ ) to $\sim 1.25 \mathrm{eV}$ (at $297 \mathrm{~K}$ ) as seen in Figure $3 \mathrm{a}$ because of the interaction with acoustic and optical phonons. ${ }^{43,44}$ Furthermore, the encapsulated InSe 
shows higher PL intensity, which further confirms the suppression of surface charge traps and defects by using the bottom hBN substrate. ${ }^{36,43}$ On measuring multiple stacks, a mix of red and blue shifts in the PL peak position is obtained when full hBN encapsulation is used (Figure S4). The ambiguity in the shift direction is attributed to multilayered structures of InSe and GaSe employed in this study. Unlike monolayers of TMDCs where a reproducible red shift due to a strong effect of hBN dielectric is observed, ${ }^{43-45}$ here, the effect is much less pronounced. While the red shift can be attributed to a stronger effect of the hBN dielectric, the blue shift may arise because of various reasons, such as the presence of shallow defect states, compositional fluctuations, and interface inhomogeneities and/or as demonstrated in TMDCs, the relative population of neutral and charged excitons. ${ }^{46-49} \mathrm{~A}$ recent study shows that in multilayered InSe, hBN initiates stronger charge confinement than $\mathrm{SiO}_{2}$, resulting in blueshifting of the optical band gap. ${ }^{50}$ Hence, the shift direction in the multilayers of InSe and GaSe is governed by several factors and cannot be associated only with the dielectric environment. Nevertheless, the superiority of full hBN encapsulation is evident in other PL features (intensity and fwhm). It is clearly seen that the PL yield is maximized when $2 \mathrm{D}$ layers are encapsulated in top and bottom hBN (Figures 3 and S4). A statistical analysis of multiple stacks (Figure S4c) confirms an overall narrowing down of the PL linewidth by $2-3$ times when 2D layers are encapsulated between top and bottom $\mathrm{hBN}$. These findings thus show that the best results are obtained when the bottom hBN layer which protects the InSe layers from the charge disorder at the $\mathrm{SiO}_{2}$ surface is used in conjunction with top encapsulation which avoids exposure of the top surface to air. ${ }^{49,51}$

The same samples are measured over time to test their longterm stability under ambient conditions. Figure $3 c$,d reveals the PL spectra measured at room temperature under normal atmospheric conditions (see Methods for detailed PL specifications) for a time period of 8 weeks. Between consecutive measurements, both samples are stored in dark under ambient conditions, allowing the ambient exposure to affect both devices in the same manner. Figure $3 \mathrm{e}$ shows that the PL peak intensity of the encapsulated InSe is intact for almost 4 weeks, after which it starts to decline gradually, whereas for the unencapsulated sample, the PL intensity has almost vanished after 2 weeks. The PL peak position, however, is not significantly affected over time (Figure 3f). Balakrishnan et al. ${ }^{28}$ have attributed the reduction in the PL intensity to the formation of $\operatorname{In}_{2} \mathrm{O}_{3}$ as InSe thin layers are exposed to air under high temperatures and focused lasers. The trend in Figure 3 indicates that the stability of the InSe layers is greatly enhanced by using hBN encapsulation which preserves InSe from the complex physical and chemical changes occurring when it comes in contact with air.

To investigate the quality of hBN encapsulated InSe FETs, electrical performance of both encapsulated and unencapsulated devices is measured in back-gate configuration. The transfer curves of both devices (Figure 4a) show an n-type FET behavior due to intrinsic doping of InSe layers as predicted previously. ${ }^{2-4}$ The room-temperature two-terminal field-effect mobility $\left(\mu_{\mathrm{FE}}\right)$ is extracted using the following equation:

$$
\mu_{\mathrm{FE}}=\frac{\mathrm{d} I_{\mathrm{DS}}}{\mathrm{d} V_{\mathrm{G}}} \cdot \frac{L}{W C_{i} V_{\mathrm{DS}}}
$$

a
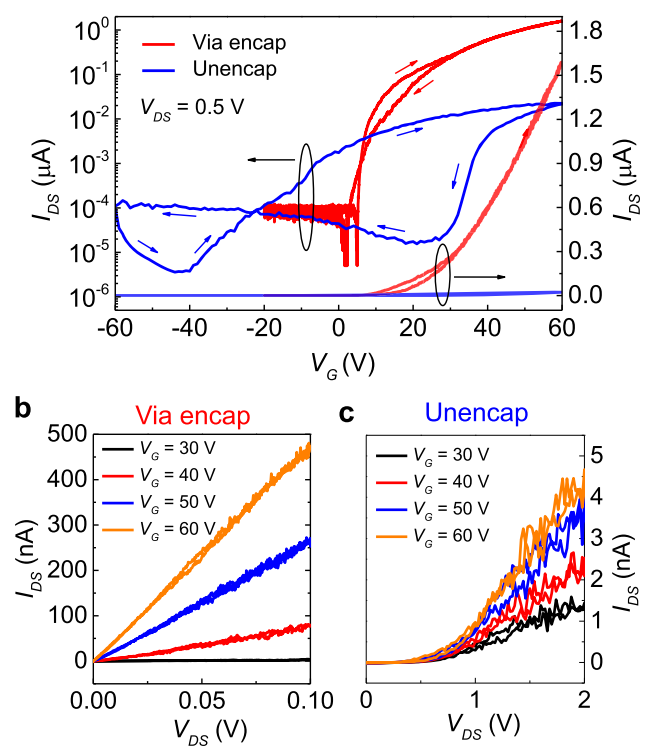

Figure 4. Electrical characterization of encapsulated InSe-via device and unencapsulated InSe device shown in Figure 1c,e, respectively. (a) Transfer characteristics (linear and semilog) of both devices at $V_{\mathrm{DS}}=0.5 \mathrm{~V}$ measured in back-gate configuration. The small arrows show the direction of the gate sweep. (b) Output characteristics of the InSe-via-encapsulated device for back-gate voltages from +30 to +60 $\mathrm{V}$ with steps of $10 \mathrm{~V}$, showing linear characteristics, indicating ohmic behavior. (c) Output characteristics of the unencapsulated InSe device measured at back-gate voltages from +30 to $+60 \mathrm{~V}$ with steps of $10 \mathrm{~V}$, showing nonlinear behavior. Note that the current level in the unencapsulated device is significantly lower than the encapsulated device even in large $V_{\mathrm{DS}}$ regimes.

where $L$ and $W$ are the length and width of the channel, $I_{\mathrm{DS}}$ and $V_{\mathrm{DS}}$ are source-drain current and voltage, $V_{\mathrm{G}}$ is the backgate voltage, and $C_{i}$ is the capacitance per unit area. $C_{i}$ estimated for the encapsulated device is $0.108 \times 10^{-7} \mathrm{~F} / \mathrm{cm}^{2}$ (full calculation provided in the Supporting Information). For the $9.6 \mathrm{~nm}$ InSe-via-encapsulated device shown in Figure 1c, the extracted $\mu_{\mathrm{FE}}$ is $30 \mathrm{~cm}^{2} \mathrm{~V}^{-1} \mathrm{~s}^{-1}$ with an on/off ratio of $10^{4}$. For other InSe-via devices with InSe thicknesses of 8-12 nm, mobility ranging $30-120 \mathrm{~cm}^{2} \mathrm{~V}^{-1} \mathrm{~s}^{-1}$ is obtained (Figure S5). In addition, the $\mathrm{hBN}$-encapsulated device shows negligible hysteresis in comparison to the unencapsulated device as well as to previously reported devices using ALD and dry oxidation as encapsulation, where substantial hysteresis was observed ${ }^{32,34}$ because of trapped charge carriers at the $\mathrm{InSe}-\mathrm{SiO}_{2}$ interface. ${ }^{2,36,52}$ Large hysteresis can give rise to further unreliability issues, for example, extraction of field-effect mobility which in a hysteric curve becomes strongly dependent on the gate sweep direction. ${ }^{53}$ For this particular unencapsulated device, we extracted a mobility of $\sim 0.47 \mathrm{~cm}^{2} \mathrm{~V}^{-1} \mathrm{~s}^{-1}$ (in forward sweep), which is the lower limit of the mobility due to large hysteresis (Figure S6b). On testing multiple unencapsulated devices, we obtained large hysteresis in all devices, poor mobility values of $\sim 1 \mathrm{~cm}^{2} \mathrm{~V}^{-1} \mathrm{~s}^{-1}$, and on/off ratios of few hundreds. The output curve of the encapsulated device (Figure $4 \mathrm{~b}$ ) reveals linear characteristics indicating the formation of ohmic contacts between the $\mathrm{Pd} / \mathrm{Au}$ metal used in the via-contact and the underlying InSe layer. However, we could not produce ohmic and reliable contacts for unencapsulated InSe FETs as revealed in Figure 4c, which might be due to degradation of InSe prior to the metal deposition, leading to tunnel barriers at the 
metal-InSe interface. ${ }^{5}$ The InSe channel area, in addition, is continuously degrading due to air exposure. Thus, as evident from our results, full hBN encapsulation is a reliable and effective passivation technique for InSe and yields a stable charge transport, greatly enhanced $\mu_{\mathrm{FE}}$ and larger on/off ratio as compared to the unencapsulated devices.

To investigate the long-term stability of InSe devices, the samples were stored and measured in air for 15 days. The transfer curve of the encapsulated device (Figure 5a) shows a
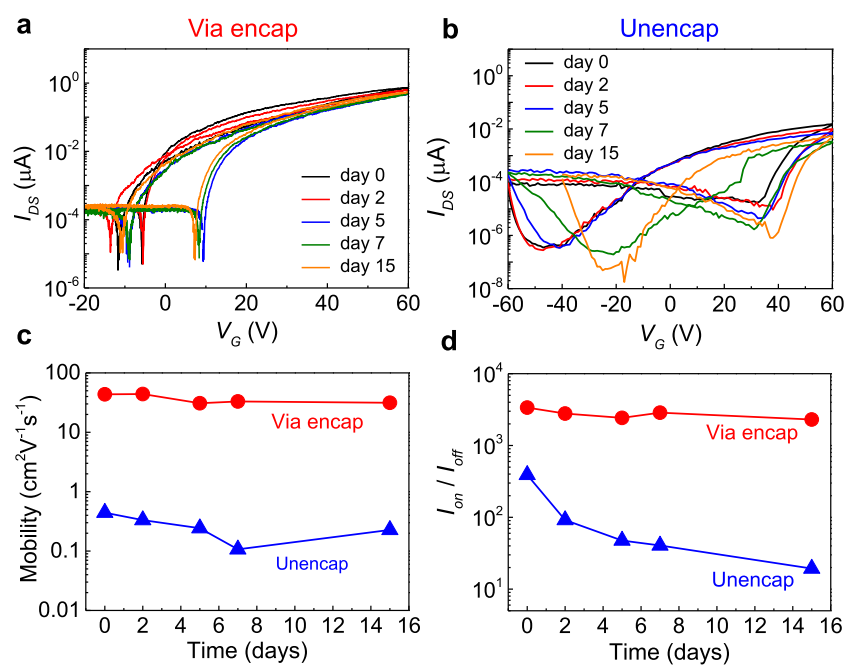

Figure 5. Transfer characteristics and FET parameters measured over time to investigate the stability of via-encapsulated and unencapsulated InSe devices. (a) Semilog transfer curve of via-encapsulated device at $V_{\mathrm{DS}}=0.5 \mathrm{~V}$ measured over 15 days. The on- and off-currents are highly reproducible with time indicating an ambient-stable device. (b) Semilog transfer curve of the unencapsulated device measured under same conditions as the encapsulated device. Large hysteresis and unstable current demonstrates unreliable device characteristics. Evolution of (c) mobility and (d) on/off ratio with time for both device configurations. Both parameters are intact for the encapsulated device, while the unencapsulated device degrades at a fast pace.

stable and consistent charge transport for over 2 weeks, whereas the unencapsulated device has a large hysteresis and low on-currents (Figure $5 \mathrm{~b}$ ). The large hysteresis and shift in the threshold voltage of the unencapsulated InSe device are attributed to the charge traps at the $\mathrm{InSe}-\mathrm{SiO}_{2}$ interface and to continuous adsorption/desorption of the gases because the samples are stored and measured in air. For the encapsulated sample, some changes in its transfer characteristics are observed after few days, which might be due to small amounts of moisture and gases getting trapped during the fabrication process. To completely mitigate these issues, fabrication of the devices can be carried out under stringent atmospheric conditions, for example, using an argon-filled glovebox instead of nitrogen. The evolution of the field-effect mobility and the on/off ratio for both devices over time is shown in Figure $5 c, d$, respectively. The unencapsulated device shows poor mobility and low on/off ratio as compared to the encapsulated device from the beginning and continues to deteriorate further with time. (Note that the unencapsulated device was fabricated and measured within a few hours from the time of exfoliation to minimize the degradation). The performance of the encapsulated device, on the other hand, stays intact throughout the measured time period. This indicates that InSe is susceptible to degradation in air and during standard process steps of device fabrication. During the whole process, InSe layers come in contact with various pre- and post-lithographic solvents, which may induce structural damage, tunnel barriers, and pinning of the Fermi level. ${ }^{38}$ The presence of tunnel barriers and Fermi level pinning results in nonohmic contacts, low on-current, and poor mobility as seen in Figure $4 \mathrm{c}$ for the unencapsulated devices. ${ }^{34,54}$ Wei et al. ${ }^{31}$ have shown that adsorption of oxygen atoms into the InSe structure causes serious hampering of the electronic properties with mobility and other FET parameters to decrease 2-3 orders in magnitude. Therefore, it is essential to passivate InSe from air and at the same time from other detrimental processes where its structural integrity can be compromised. In this regard, the top and bottom $\mathrm{hBN}$ encapsulation scheme is extremely useful as it protects InSe from various forms of degradation and disorder and allows us to study its intrinsic properties.

Subsequently, we integrated the full hBN encapsulation technique into GaSe, another emerging 2D material belonging to the same family as InSe but far more sensitive under ambient conditions. The effectiveness of hBN encapsulation in suppressing GaSe degradation is supported by low-temperature PL measurements (Figure 6a,b) which show a significant
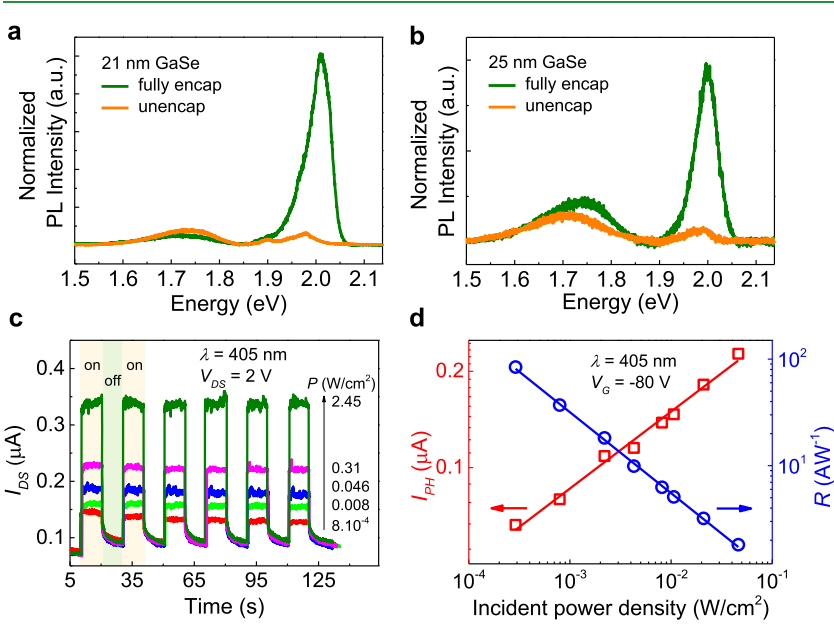

Figure 6. Optical characterization of fully encapsulated GaSe devices. PL spectra measured at $4.2 \mathrm{~K}$ for GaSe layers (a) $21 \mathrm{~nm}$ thick and (b) $25 \mathrm{~nm}$ thick. Fully encapsulated GaSe shows intense PL peaks at $\sim 2.0$ $\mathrm{eV}$, while unencapsulated GaSe shows PL reduction due to fast degradation. (c) Photoswitching response obtained for pulsed illumination of $405 \mathrm{~nm}$ at $V_{\mathrm{G}}=-80 \mathrm{~V}, V_{\mathrm{DS}}=2 \mathrm{~V}$, and various laser power densities. (d) Photocurrent (red open squares) and responsivity (blue open dots) as a function of incident power density at $V_{\mathrm{G}}=-80 \mathrm{~V}$ and $V_{\mathrm{DS}}=2 \mathrm{~V}$. Red and blue straight lines are obtained by fitting the measured data.

improvement in the spectra of the encapsulated GaSe as compared to the unencapsulated layer. On measuring various thicknesses of GaSe layers, we observed 10 times higher PL peak intensity when it is encapsulated in top and bottom hBN, whereas unencapsulated GaSe shows significant PL reduction within $3 \mathrm{~h}$ from the time of exfoliation.

Because GaSe finds a majority of its applications in optics, the large band gap of hBN $(\sim 5.7 \mathrm{eV})^{55}$ is highly advantageous, as it stays transparent for the wavelengths where GaSe is optically active. The photoresponse obtained for the GaSe-via device under global illumination by a blue laser $(405 \mathrm{~nm})$ at various power settings is shown in Figure $6 \mathrm{c}, \mathrm{d}$. As the device is illuminated by higher laser power, $I_{\mathrm{DS}}$ increases monotonically 
because of the increased number of electron-hole pairs generated. In response to pulsed illumination at $405 \mathrm{~nm}$, the device shows a photoswitching behavior and maintains longterm stability of its photoresponse (Figure 6c). The rise in current from off- to steady-state on light illumination occurs within a $20 \mathrm{~ms}$ time interval, which is the resolution of the measurement setup. We believe that the photoresponse time should be less than $20 \mathrm{~ms}$, which is comparable to the response times of $10-20 \mathrm{~ms}$ reported previously with top gold contacts. $^{22,37}$ The photocurrent $\left(I_{\mathrm{PH}}=I_{\text {light }}-I_{\text {dark }}\right)$ plotted in Figure 6d increases with increasing incident power densities. Responsivity, defined as the amount of the photocurrent generated per unit incident illumination and calculated as the ratio of the photocurrent density to the incident power density $\left(R=I_{\mathrm{PH}} / P A ; P\right.$ is incident power density; $A$ is exposed device area) ${ }^{56}$ is found to be decreasing as the incident power increases. At an incident power density of $0.3 \mathrm{~mW} / \mathrm{cm}^{2}\left(V_{\mathrm{G}}=\right.$ $-80 \mathrm{~V}$ and $\left.V_{\mathrm{DS}}=2 \mathrm{~V}\right)$, we achieved a responsivity of $84.2 \mathrm{~A}$ $\mathrm{W}^{-1}$, which is a major improvement over top-encapsulated GaSe photodetectors reported previously. ${ }^{21}$

\section{CONCLUSIONS}

The sensitivity of few-layered InSe and GaSe toward air, bottom dielectrics, and lithography processes makes the assessment of their intrinsic properties difficult. We demonstrate that in order to obtain high-quality and stable devices, their full encapsulation in hBN layers is extremely advantageous as it protects the $2 \mathrm{D}$ layers from ambient degradation as well as from the charge disorder at the $\mathrm{SiO}_{2}$ surface. The fully encapsulated InSe and $\mathrm{GaSe}$ devices show greatly enhanced and ambient-stable performance as compared to their unencapsulated counterparts. $\mathrm{hBN}$, because of its inertness, atomically flat surface, and absence of charge traps, is an optimal encapsulation for fabricating reliable InSe- and GaSebased devices. In the future, this technique can be applied to other sensitive $2 \mathrm{D}$ materials which might then supersede existing materials in terms of properties and performance.

\section{METHODS}

Bulk crystals of InSe (3R phase with a purity of 99.999\%) and GaSe (2H phase with a purity of $99.995 \%$ ) are bought commercially from 2dSemiconductors and hqgraphene, respectively. The crystals are mechanically exfoliated using the adhesive tape (model BT-130E-SL bought from TELTEC GmBH) inside a nitrogen-filled glovebox and stacked between top and bottom hBN using the "vdW transfer" technique, described in detail in the Supporting Information. To fabricate via-contacts, holes are etched into $\mathrm{hBN}$ flakes using reactiveion etching (Oxford RIE) with a gas mixture of $\mathrm{SF}_{6}$ and $\mathrm{O}_{2}$. The holes are deposited with Pd: $20 \mathrm{~nm} / \mathrm{Au}: 40 \mathrm{~nm}$ using an e-beam evaporator. For lift-off process, the samples are dipped into acetone for at least $2 \mathrm{~h}$ followed by rinsing in isopropanol and nitrogen blow drying. The metal extensions to the $\mathrm{hBN} / \mathrm{InSe} /$ via-hBN stack are fabricated by using e-beam lithography under a poly(methyl methacrylate) e-beam resist mask followed by metal deposition (Cr: $2 \mathrm{~nm} / \mathrm{Pd}$ : $20 \mathrm{~nm} / \mathrm{Au}$ : $40 \mathrm{~nm}$ ) and standard lift-off process as described above. EDS is carried out at $20 \mathrm{kV}$ by means of a conventional $\mathrm{Si}(\mathrm{Li})$ detector with the S-UTW window (Oxford Instruments) attached to a scanning electron microscope. EDS spectra are obtained with the INCA software by scanning a specimen area of about $3 \times 5 \mu \mathrm{m}^{2}$ for a duration of $1 \mathrm{~h}$ and acquiring the data with an energy dispersion of 10 $\mathrm{eV} /$ channel in an energy range of $0-20 \mathrm{keV}$. Low-temperature microPL measurements are carried out using a LHe cryostat system. The excitation pump is a $\mathrm{cw}$ frequency-doubled $\mathrm{Nd} / \mathrm{YAG}$ laser at a wavelength of $532 \mathrm{~nm}$. The power is $10 \mu \mathrm{W}$ focused on a spot diameter of $3 \mu \mathrm{m}$. The spectra are captured on a liquid nitrogen- cooled silicon CCD deep-depletion camera after being dispersed in a 300 lines/mm grating spectrometer. Electrical measurements are performed using a parameter analyzer (Agilent, 4155C) and photoresponse using Lake Shore probe station (Model CPX-VF) equipped with a cw blue laser diode at $405 \mathrm{~nm}$ wavelength. All electrical and photocurrent measurements are carried out in air and at room temperature.

\section{ASSOCIATED CONTENT}

\section{Supporting Information}

The Supporting Information is available free of charge on the ACS Publications website at DOI: 10.1021/acsami.9b13442.

Details on vdW heterostructure fabrication, EDS and Raman characterization of GaSe, low-temperature micro-PL measurements on InSe-hBN stacks, and electrical characterization of other InSe-via devices (PDF)

\section{AUTHOR INFORMATION}

\section{Corresponding Authors}

*E-mail: jh2228@columbia.edu (J.C.H.).

*E-mail: a.erbe@hzdr.de (A.E.).

ORCID $\odot$

Himani Arora: 0000-0003-3318-9877

Author Contributions

H.A. and Y.J. contributed equally. H.A. exfoliated InSe and GaSe from bulk crystals and fabricated vdW heterostructures of hBN and InSe or GaSe. Y.J. fabricated via-contacts and prepared devices by electron-beam lithography. T.V., H.S., and H.A. performed PL measurements. H.A. measured charge transport and photoresponse in the devices. K.W and T.T. provided hBN crystals. All authors discussed the results and participated in the preparation of the manuscript. J.C.H. and A.E. supervised the project.

\section{Notes}

The authors declare no competing financial interest.

\section{ACKNOWLEDGMENTS}

We thank D. Rhodes, N. R. Finney, and G. Arefe for their help in device fabrication and subject matter discussion and $\mathrm{F}$. Kilibarda and J. Franke for drawing the schematics. Growth of hBN crystals was supported by the Elemental Strategy Initiative conducted by the MEXT, Japan, A3 Foresight by JSPS and the CREST (JPMJCR15F3), JST. This work was kindly supported by the Initiative and Networking Fund of the Helmholtz Association of German Research Centers through the International Helmholtz Research School for Nanoelectronic Networks, IHRS NANONET (VH-KO-606) and INSPIRE grant from Center of Advancing Electronics Dresden (cfaed).

\section{REFERENCES}

(1) Bandurin, D. A.; Tyurnina, A. V.; Yu, G. L.; Mishchenko, A.; Zólyomi, V.; Morozov, S. V.; Kumar, R. K.; Gorbachev, R. V.; Kudrynskyi, Z. R.; Pezzini, S.; Kovalyuk, Z. D.; Zeitler, U.; Novoselov, K. S.; Patanè, A.; Eaves, L.; Grigorieva, I. V.; Fal'Ko, V. I.; Geim, A. K.; Cao, Y. High Electron Mobility, Quantum Hall Effect and Anomalous Optical Response in Atomically Thin InSe. Nat. Nanotechnol. 2017, 12, 223-227.

(2) Sucharitakul, S.; Goble, N. J.; Kumar, U. R.; Sankar, R.; Bogorad, Z. A.; Chou, F.-C.; Chen, Y.-T.; Gao, X. P. A. Intrinsic Electron Mobility Exceeding $1000 \mathrm{~cm}^{2} /(\mathrm{Vs})$ in Multilayer InSe FETs. Nano Lett. 2015, 15, 3815-3819. 
(3) Feng, W.; Zheng, W.; Cao, W.; Hu, P. Back Gated Multilayer InSe Transistors with Enhanced Carrier Mobilities Via the Suppression of Carrier Scattering from a Dielectric Interface. Adv. Mater. 2014, 26, 6587-6593.

(4) Feng, W.; Zhou, X.; Tian, W. Q.; Zheng, W.; Hu, P. Performance Improvement of Multilayer InSe Transistors with Optimized Metal Contacts. Phys. Chem. Chem. Phys. 2015, 17, 3653-3658.

(5) Yang, H.-W.; Hsieh, H.-F.; Chen, R.-S.; Ho, C.-H.; Lee, K.-Y.; Chao, L.-C. Ultraefficient Ultraviolet and Visible Light Sensing and Ohmic Contacts in High-Mobility InSe Nanoflake Photodetectors Fabricated by the Focused Ion Beam Technique. ACS Appl. Mater. Interfaces 2018, 10, 5740-5749.

(6) Zheng, L.; Yu, P.; Hu, K.; Teng, F.; Chen, H.; Fang, X. ScalableProduction, Self-Powered $\mathrm{TiO}_{2}$ Nanowell-Organic Hybrid UV Photodetectors with Tunable Performances. ACS Appl. Mater. Interfaces 2016, 8, 33924-33932.

(7) Tamalampudi, S. R.; Lu, Y.-Y.; Kumar, U.R.; Sankar, R.; Liao, C.D.; Moorthy, B.K.; Cheng, C.-H.; Chou, F. C.; Chen, Y.-T. High Performance and Bendable Few-Layered InSe Photodetectors with Broad Spectral Response. Nano Lett. 2014, 14, 2800-2806.

(8) Abderrahmane, A.; Jung, P.-G.; Kim, N.-H.; Ko, P. J.; Sandhu, A. Gate-Tunable Optoelectronic Properties of a Nano-Layered GaSe Photodetector. Opt. Mater. Express 2017, 7, 587-592.

(9) Yang, Z.; Jie, W.; Mak, C.-H.; Lin, S.; Lin, H.; Yang, X.; Yan, F.; Lau, S. P.; Hao, J. Wafer-Scale Synthesis of High-Quality Semiconducting Two-Dimensional Layered InSe with Broadband Photoresponse. ACS Nano 2017, 11, 4225-4236.

(10) Yang, W.; Hu, K.; Teng, F.; Weng, J.; Zhang, Y.; Fang, X. HighPerformance Silicon-Compatible Large-Area UV-to-Visible Broadband Photodetector Based on Integrated Lattice-Matched Type II Se/ n-Si Heterojunctions. Nano Lett. 2018, 18, 4697-4703.

(11) Late, D. J.; Liu, B.; Luo, J.; Yan, A.; Matte, H. S. S. R.; Grayson, M.; Rao, C. N. R.; Dravid, V. P. GaS and GaSe Ultrathin Layer Transistors. Adv. Mater. 2012, 24, 3549-3554.

(12) Savchyn, V. P.; Kytsai, V. B. Photoelectric Properties of Heterostructures Based on Thermo-Oxidated GaSe and InSe Crystals. Thin Solid Films 2000, 361-362, 123-125.

(13) Li, Z.; Yang, Y.; Wang, X.; Shi, W.; Xue, D.-J.; Hu, J.-S. ThreeDimensional Optical Anisotropy of Low-Symmetry Layered GeS. ACS Appl. Mater. Interfaces 2019, 11, 24247-24253.

(14) Shi, W.; Ding, Y. J. A Monochromatic and High-Power Terahertz Source Tunable in the Ranges of 2.7-38.4 and 58.2-3540 $\mu \mathrm{m}$ for Variety of Potential Applications. Appl. Phys. Lett. 2004, 84, $1635-1637$.

(15) Kuroda, N.; Nishina, Y. Resonance Raman Scattering Study on Exciton and Polaron Anisotropies in InSe. Solid State Commun. 1980, 34, 481-484.

(16) Mudd, G. W.; Svatek, S. A.; Ren, T.; Patanè, A.; Makarovsky, O.; Eaves, L.; Beton, P. H.; Kovalyuk, Z. D.; Lashkarev, G. V.; Kudrynskyi, Z. R.; Dmitriev, A. I. Tuning the Bandgap of Exfoliated InSe Nanosheets by Quantum Confinement. Adv. Mater. 2013, 25, 5714-5718.

(17) Le Toullec, R.; Balkanski, M.; Besson, J. M.; Kuhn, A. Optical Absorption Edge of a New GaSe Polytype. Phys. Lett. A 1975, 55, 245-246.

(18) Bube, R. H.; Lind, E. L. Photoconductivity of Gallium Selenide Crystals. Phys. Rev. 1959, 115, 1159-1164.

(19) Minder, R.; Ottaviani, G.; Canali, C. Charge Transport in Layer Semiconductors. J. Phys. Chem. Solids 1976, 37, 417-424.

(20) Jie, W.; Hao, J. Two-Dimensional Layered Gallium Selenide: Preparation, Properties, and Applications. In Advanced 2D Materials; Tiwari, A., Syvajarvi, M., Eds.; John Wiley \& Sons: Hoboken, NJ, USA, 2016; Vol. 1, pp 1-36.

(21) Zhao, Q.; Frisenda, R.; Gant, P.; Perez de Lara, D.; Munuera, C.; Garcia-Hernandez, M.; Niu, Y.; Wang, T.; Jie, W.; CastellanosGomez, A. Toward Air Stability of Thin GaSe Devices: Avoiding Environmental and Laser-Induced Degradation by Encapsulation. Adv. Funct. Mater. 2018, 28, 1805304.
(22) Hu, P.; Wen, Z.; Wang, L.; Tan, P.; Xiao, K. Synthesis of FewLayer GaSe Nanosheets for High Performance Photodetectors. ACS Nano 2012, 6, 5988-5994.

(23) Tonndorf, P.; Schwarz, S.; Kern, J.; Niehues, I.; Del PozoZamudio, O.; Dmitriev, A. I.; Bakhtinov, A. P.; Borisenko, D. N.; Kolesnikov, N. N.; Tartakovskii, A. I.; Michaelis de Vasconcellos, S.; Bratschitsch, R. Single-Photon Emitters in GaSe. 2D Mater. 2017, 4, 021010.

(24) Jie, W.; Chen, X.; Li, D.; Xie, L.; Hui, Y. Y.; Lau, S. P.; Cui, X.; Hao, J. Layer-Dependent Nonlinear Optical Properties and Stability of Non-Centrosymmetric Modification in Few-Layer GaSe Sheets. Angew. Chem. Int. Ed. 2015, 54, 1185-1189.

(25) Rahaman, M.; Rodriguez, R. D.; Monecke, M.; Lopez-Rivera, S. A.; Zahn, D. R. T. GaSe Oxidation in Air: from Bulk to Monolayers. Semicond. Sci. Technol. 2017, 32, 105004.

(26) Del Pozo-Zamudio, O.; Schwarz, S.; Klein, J.; Schofield, R. C.; Chekhovich, E. A.; Ceylan, O.; Margapoti, E.; Dmitriev, A. I.; Lashkarev, G. V.; Borisenko, D. N.; Kolesnikov, N. N.; Finley, J. J.; Tartakovskii, A. I. Photoluminescence and Raman Investigation of Stability of InSe and GaSe Thin Films, 2015, arXiv: 1506.05619 [cond-mat. mes-hall].

(27) Bergeron, A.; Ibrahim, J.; Leonelli, R.; Francoeur, S. Oxidation Dynamics of Ultrathin GaSe Probed Through Raman Spectroscopy. Appl. Phys. Lett. 2017, 110, 241901.

(28) Balakrishnan, N.; Kudrynskyi, Z. R.; Smith, E. F.; Fay, M. W.; Makarovsky, O.; Kovalyuk, Z. D.; Eaves, L.; Beton, P. H.; Patanè, A. Engineering P-N Junctions and Bandgap Tuning of InSe Nanolayers by Controlled Oxidation. 2D Mater. 2017, 4, 025043.

(29) Politano, A.; Chiarello, G.; Samnakay, R.; Liu, G.; Gürbulak, B.; Duman, S.; Balandin, A. A.; Boukhvalov, D. W. The Influence of Chemical Reactivity of Surface Defects on Ambient-Stable InSe-Based Nanodevices. Nanoscale 2016, 8, 8474-8479.

(30) Shi, L.; Zhou, Q.; Zhao, Y.; Ouyang, Y.; Ling, C.; Li, Q.; Wang, J. Oxidation Mechanism and Protection Strategy of Ultrathin Indium Selenide: Insight from Theory. J. Phys. Chem. Lett. 2017, 8, 43684373.

(31) Wei, X.; Dong, C.; Xu, A.; Li, X.; Macdonald, D. D. OxygenInduced Degradation of the Electronic Properties of Thin-Layer InSe. Phys. Chem. Chem. Phys. 2018, 20, 2238-2250.

(32) Ho, P.-H.; Chang, Y.-R.; Chu, Y.-C.; Li, M.-K.; Tsai, C.-A.; Wang, W.-H.; Ho, C.-H.; Chen, C.-W.; Chiu, P.-W. High-Mobility InSe Transistors: the Role of Surface Oxides. ACS Nano 2017, 11, $7362-7370$

(33) Arora, H.; Schönherr, T.; Erbe, A. Electrical Characterization of Two-Dimensional Materials and Their Heterostructures. IOP Conf. Ser. Mater. Sci. Eng. 2017, 198, 012002.

(34) Wells, S. A.; Henning, A.; Gish, J. T.; Sangwan, V. K.; Lauhon, L. J.; Hersam, M. C. Supressing Ambient Degradation of Exfoliated InSe Nanosheet Devices Via Seeded Atomic Layer Deposition Encapsulation. Nano Lett. 2018, 18, 7876-7882.

(35) Dean, C. R.; Young, A. F.; Meric, I.; Lee, C.; Wang, L.; Sorgenfrei, S.; Watanabe, K.; Taniguchi, T.; Kim, P.; Shepard, K. L.; Hone, J. Boron Nitride Substrates for High-Quality Graphene Electronics. Nat. Nanotechnol. 2010, 5, 722-726.

(36) Lee, G.-H.; Cui, X.; Kim, Y. D.; Arefe, G.; Zhang, X.; Lee, C.H.; Ye, F.; Watanabe, K.; Taniguchi, T.; Kim, P.; Hone, J. Highly Stable, Dual-Gated $\mathrm{MoS}_{2}$ Transistors Encapsulated by Hexagonal Boron Nitride with Gate-Controllable Contact, Resistance, and Threshold Voltage. ACS Nano 2015, 9, 7019-7026.

(37) Cao, Y.; Cai, K.; Hu, P.; Zhao, L.; Yan, T.; Luo, W.; Zhang, X.; Wu, X.; Wang, K.; Zheng, H. Strong Enhancement of Photoresponsivity with Shrinking the Electrodes Spacing in Few Layer GaSe Photodetectors. Sci. Rep. 2015, 5, 8130.

(38) Telford, E. J.; Benyamini, A.; Rhodes, D.; Wang, D.; Jung, Y.; Zangiabadi, A.; Watanabe, K.; Taniguchi, T.; Jia, S.; Barmak, K.; Pasupathy, A. N.; Dean, C. R.; Hone, J. Via Method for Lithography Free Contact and Preservation of 2D Materials. Nano Lett. 2018, 18, $1416-1420$. 
(39) Wang, L.; Meric, I.; Huang, P. Y.; Gao, Q.; Gao, Y.; Tran, H.; Taniguchi, T.; Watanabe, K.; Campos, L. M.; Muller, D. A.; Guo, J.; Kim, P.; Hone, J.; Shepard, K. L.; Dean, C. R. One-Dimensional Electrical Contact to a Two-Dimensional Material. Science 2013, 342, 614-617.

(40) Lei, S.; Ge, L.; Najmaei, S.; George, A.; Kappera, R.; Lou, J.; Chhowalla, M.; Yamaguchi, H.; Gupta, G.; Vajtai, R.; Mohite, A. D.; Ajayan, P. M. Evolution of the Electronic Band Structure and Efficient Photo-Detection in Atomic Layers of InSe. ACS Nano 2014, 8, 12631272.

(41) Li, X.; Lin, M.-W.; Puretzky, A. A.; Idrobo, J. C.; Ma, C.; Chi, M.; Yoon, M.; Rouleau, C. M.; Kravchenko, I. I.; Geohegan, D. B.; Xiao, K. Controlled Vapor Phase Growth of Single Crystalline, TwoDimensional GaSe Crystals with High Photoresponse. Sci. Rep. 2015, 4, 5497.

(42) Zhang, X.; Tan, Q.-H.; Wu, J.-B.; Shi, W.; Tan, P.-H. Review on the Raman Spectroscopy of Different Types of Layered Materials. Nanoscale 2016, 8, 6435-6450.

(43) Cadiz, F.; Courtade, E.; Robert, C.; Wang, G.; Shen, Y.; Cai, H.; Taniguchi, T.; Watanabe, K.; Carrere, H.; Lagarde, D.; Manca, M.; Amand, T.; Renucci, P.; Tongay, S.; Marie, X.; Urbaszek, B. Excitonic Linewidth Approaching the Homogeneous Limit in $\mathrm{MoS}_{2-}$ Based Van Der Waals Heterostructures. Phys. Rev. X 2017, 7, 021026.

(44) Manca, M.; Glazov, M. M.; Robert, C.; Cadiz, F.; Taniguchi, T.; Watanabe, K.; Courtade, E.; Amand, T.; Renucci, P.; Marie, X.; Wang, G.; Urbaszek, B. Enabling Valley Selective Exciton Scattering in Monolayer $\mathrm{WSe}_{2}$ Through Upconversion. Nat. Commun. 2017, 8, 14927.

(45) Ajayi, O. A.; Ardelean, J. V.; Shepard, G. D.; Wang, J.; Antony, A.; Taniguchi, T.; Watanabe, K.; Heinz, T. F.; Strauf, S.; Zhu, X.-Y.; Hone, J. C. Approaching the Intrinsic Photoluminescence Linewidth in Transition Metal Dichalcogenide Monolayers. 2D Mater 2017, 4, 031011.

(46) Eliseev, P. G.; Perlin, P.; Lee, J.; Osiński, M. "Blue" Temperature-Induced Shift and Band-Tail Emission in InGaNBased Light Sources. Appl. Phys. Lett. 1997, 71, 569-571.

(47) Cho, Y.-H.; Gainer, G. H.; Fischer, A. J.; Song, J. J.; Keller, S.; Mishra, U. K.; DenBaars, S. P. "S-Shaped" Temperature-Dependent Emission Shift and Carrier Dynamics in InGaN/GaN Multiple Quantum Wells. Appl. Phys. Lett. 1998, 73, 1370-1372.

(48) Buscema, M.; Steele, G. A.; van der Zant, H. S. J.; CastellanosGomez, A. The Effect of the Substrate on the Raman and Photoluminescence Emission of Single-Layer $\mathrm{MoS}_{2}$. Nano Res. 2014, 7, 561-571.

(49) Wierzbowski, J.; Klein, J.; Sigger, F.; Straubinger, C.; Kremser, M.; Taniguchi, T.; Watanabe, K.; Wurstbauer, U.; Holleitner, A. W.; Kaniber, M.; Müller, K.; Finley, J. J. Direct Exciton Emission from Atomically Thin Transition Metal Dichalcogenide Heterostructures near the Lifetime Limit. Sci. Rep. 2017, 7, 12383.

(50) Makkawi, O.; Qiu, Y.; Feng, W.; Hu, P. A. The Modulation of Photoluminscences Band Gap of Two-Dimensional InSe Nanosheets on h-BN Substrate. J. Nanosci. Nanotechnol. 2016, 16, 9813-9819.

(51) Venanzi, T.; Arora, H.; Erbe, A.; Pashkin, A.; Winnerl, S.; Helm, M.; Schneider, H. Exciton Localization in $\mathrm{MoSe}_{2}$ Monolayers Induced by Adsorbed Gas Molecules. Appl. Phys. Lett. 2019, 114, 172106.

(52) Doganov, R. A.; O’Farrell, E. C. T.; Koenig, S. P.; Yeo, Y.; Ziletti, A.; Carvalho, A.; Campbell, D. K.; Coker, D. F.; Watanabe, K.; Taniguchi, T.; Castro Neto, A. H.; Özyilmaz, B. Transport Properties of Pristine Few-Layer Black Phosphorus by Van Der Waals Passivation in an Inert Atmosphere. Nat. Commun. 2015, 6, 6647.

(53) Egginger, M.; Bauer, S.; Schwödiauer, R.; Neugebauer, H.; Sariciftci, N. S. Current Versus Gate Voltage Hysteresis in Organic Field Effect Transistors. Monatsh. Chem. 2009, 140, 735-750.

(54) Xiao, K. J.; Carvalho, A.; Castro Neto, A. H. Defects and Oxidation Resilience in InSe. Phys. Rev. B 2017, 96, 054112.

(55) Watanabe, K.; Taniguchi, T.; Kanda, H. Direct-Bandgap Properties and Evidence for Ultraviolet Lasing of Hexagonal Boron Nitride Single Crystal. Nat. Mater. 2004, 3, 404-409.
(56) Arora, H.; Malinowski, P. E.; Chasin, A.; Cheyns, D.; Steudel, S.; Schols, S.; Heremans, P. Amorphous Indium-Gallium-Zinc-Oxide as Electron Transport Layer in Organic Photodetectors. Appl. Phys. Lett. 2015, 106, 143301. 